\title{
Nutzung einer terminorientierten Besamung beim Rind auf der Grundlage einer Prostaglandin - GnRH-Behandlung
}

\begin{abstract}
Title of the paper: Practical application of timed artificial insemination in cattle using a GnRH and Prostaglandin treatment

Three experiments were conducted to establish a regime of oestrous synchronization and artificial insemination at a predetermined time in dairy cattle. In experiment 1 , dairy heifers $(n=43)$ were synchronized by administering $\mathrm{PGF}_{2 \alpha}$ on day 5, 8, 11 or 14 and the LH surge was recorded. Release of the LH surge ranged from $57.0 \pm 8.6 \mathrm{~h}$ to $76.3 \pm 10.0 \mathrm{~h}$ depending upon the day of induced luteolysis. The release of LH was followed by ovulations. The day of $\mathrm{PGF}_{2 \alpha}$ administration did not affect the interval from $\mathrm{LH}$ peak to ovulation. In experiment 2, heifers $(n=30)$ were synchronized on days 8,11 or 14 of the oestrous cycle. Animals received an injection of GnRH $65 \mathrm{~h}$ after induction of luteolysis. Uniform intervals from $\mathrm{PGF}_{2 \alpha}$ application to LH surge were observed in animals given $\mathrm{PGF}_{2 \alpha}$ on day 11 and 14. Variation for this parameter decreased also in the group of animals where luteolysis was induced on day 8. There were no significant differences due to treatment in the mean interval from LH-peak to ovulation.

In experiment 3, fixed time insemination was tested on the basis of data from Experiments 1 and 2 . Synchronized heifers and high yielding cows received GnRH 65 hours after PGF $_{2 \alpha}$ administererd between d 8 and 14 of the oestrous cycle. Artificial insemination was carried out $13 \mathrm{~h}$ after GnRH application. Pregnancy rates show, that fixed time artificial insemination, independent of the recorded onset of estrus can result in high pregnancy rates. Pregnancy rates in animals at different locations were higher after induction of ovulation and timed artificial insemination (79.0, 76.0 and 47.1, $53.3 \%$ ) than in control groups with insemination after detected estrus (69.8, 63.2 and 38.6, 45.0\%).
\end{abstract}

Key Words: cattle, $\mathrm{PGF}_{2 \alpha}$, GnRH, LH surge, ovulation, timed insemination

\section{Zusammenfassung}

Prostaglandin $\mathrm{F}_{2 \mathrm{a}}\left(\mathrm{PGF}_{2 \mathrm{a}}\right)$, verabreicht zwischen dem 5. bis 14. Zyklustag, löste bei > $90 \%$ der behandelten Tiere Östrussymptome aus. Mit zunehmendem Zeitintervall zwischen der letzten Brunst und der induzierten Luteolyse erhöhte sich der Anteil Tiere, der mit einer vollständigen Luteolyse und einem endogenen LH-Peak, als wesentliche Voraussetzung für nachfolgende Ovulationen, auf die Behandlung reagierte. Das Zeitintervall von der $\mathrm{PGF}_{2 \mathrm{a}}$-Applikation bis zum LH-Peak bzw. bis zur Ovulation wurde z. T. signifikant vom Zyklustag, an dem $\mathrm{PGF}_{2 \mathrm{a}}$ verabreicht wurde, beeinflusst. Eine Verabreichung von PGF $_{2 \mathrm{a}}$ an den Zyklustagen 5, 8, 11 bzw. 14 löste den endogenen LH-Peak nach durchschnittlich 57,0 h, 62,7 h, 76,3 h bzw. 69,5 h aus. Durchschnittlich 22 h nach dem LH-Peak wurden die Ovulationen festgestellt. Für die Auslösung fertiler Brunsten sollte PGF 2a $_{\text {a }}$ nicht vor dem 8. Zyklustag angewandt werden. GnRH, in zeitlicher Nähe zum endogenen LH-Peak injiziert, konnte den LH-Peak und die nachfolgenden Ovulationen in Tiergruppen synchronisieren und damit auch die Varianz für beide Parameter einschränken. Auf der Basis terminierter Ovulationszeiträume wurden nach terminorientierter Besamung höhere Trächtigkeitsraten als in den Kontrollgruppen erreicht. Der Abstand von 13 h zwischen der GnRH-Injektion und der künstlichen Besamung erwies sich unter Beachtung der Kapazitationszeit für Bullenspermien und der Restvarianz für den Parameter Ovulationszeitraum als günstig für die Erzielung hoher Befruchtungs- und Trächtigkeitsraten. 
BECKER et al.: Nutzung einer terminorientierten Besamung beim Rind auf der Grundlage einer Prostaglandin - GnRH-Behandlung

\section{Einleitung}

Im Fruchtbarkeitsmanagement von Milchrindherden besitzt die Künstliche Besamung (KB) eine herausragende Bedeutung. Sehr bedeutsam für den Besamungserfolg ist die Wahl des Zeitpunktes für die Insemination. Gegenwärtig werden in Europa nahezu alle Rinder, die einer Besamung zugeführt werden, brunstorientiert besamt. Die Trächtigkeitsergebnisse sind trotz allgemein gleicher Vorgehensweise mit erheblichen Variationen behaftet. Die Ursachen dafür liegen sowohl im Management als auch im biologischen Bereich. Die wichtigste Ursache besteht in der Schwierigkeit, den optimalen Besamungszeitpunkt festzulegen. Im Regelfall sollte die Besamung 8 - 12 h nach Brunstbeginn erfolgen. Da der Brunstbeginn jedoch subjektiv festgelegt wird, und diese Festlegung von einer Vielzahl von Faktoren abhängt, kann der Brunstbeginn nicht als objektives Kriterium für einen optimalen Abstand zwischen Insemination und Ovulation gewertet werden.

Der einzige objektive zeitliche Bezugspunkt - der LH-Peak - ist dem Anwender jedoch im Regelfall nicht zugänglich. Um diesen Bezugspunkt nutzen zu können, sind Kenntnisse über die Beeinflussung der Follikelentwicklung und die Luteolyse essenziell. Mit einer Vielzahl unterschiedlicher Synchronisationsregime (TWAGIRAMUNGU et al., 1995; SCHMITT et al., 1996; AMBROSE et al., 2001; RAJAMAHENDRAN et al., 2001) wurde insbesondere im nordamerikanischen Raum versucht, die Ovulation so zu terminieren, dass ein bis zwei zeitlich festgelegte Besamungen für eine Befruchtung der freigesetzten Eizelle ausreichen.

Auf der Grundlage ultrasonographischer Untersuchungen wurde Mitte der 90er Jahre versucht, Synchronisationsregime zu entwickeln, welche die Elimination des dominaten Follikels bewirken und eine neue Follikelreifungswelle initiieren sollen. In diesem Zusammenhang wurde eine Vielzahl (SCHMITT et al., 1996; PURSLEY et al., 1997; CARTMILL et al., 2001; MOREIRA et al., 2000; PETERS and PURSLEY, 2001) von zum Teil widersprüchlichen Ergebnissen zum OVSYNC-Verfahren veröffentlicht. In Untersuchungen von BOJWANI et al. (2001) wurde auf die biologischen Ursachen der zum Teil unbefriedigenden Trächtigkeitsergebnisse hingewiesen.

Ziel der nachfolgenden Untersuchungen war es, auf der Basis von Untersuchungen zur Luteolyse und zum Ovulationsverhalten nach einer Prostaglandininjektion in Kombination mit einer GnRH-Applikation ein Synchronisationregime zu entwickeln, welches eine terminorientierte Besamung unabhängig von der Erfassung der Brunstbeginns ermöglicht und hohe Trächtigkeitsraten garantiert.

\section{Material und Methoden}

An insgesamt 314 Färsen wurden in drei Abschnitten Untersuchungen zur Luteolyse und Ovulation im Anschluss an eine Brunst- bzw. Brunst- und Ovulationsinduktion an den Zyklustagen 5, 8, 11 oder 14 durchgeführt. Ein Teil der Tiere wurde in Feldexperimenten terminorientiert besamt. Als Kontrolle dienten spontanbrünstige Tiere.

Insgesamt 117 Hochleistungskühen mit einer durchschnittlichen Milchleistung von $9200 \pm 324 \mathrm{~kg}$ wurden terminorientiert besamt und die erreichten Ergebnisse mit denen nach brunstorientierter Besamung verglichen.

Für die Besamungen wurde handelsübliches, kryokonserviertes Sperma von verschiedenen Prüf- und zuchtwertgeprüften Bullen genutzt. 


\section{Experiment 1}

Insgesamt 43 Jungrinder der Rasse SMR wurde an den Zyklustagen 5, 8, 11 und 14 das Prostaglandinpräparat Cloprostenol forte ${ }^{\circledR}$ (animedica, Deutschland) in einer Dosis von 0,5 mg/Tier verabreicht. Im Zeitraum von 49 bis 85 h nach der Luteolyseinduktion wurde über Venenverweilkatheter im 2 h-Rhythmus Blutproben für die Bestimmung der LH-Konzentration entnommen. Der Verlauf der Luteolyse wurde durch Progesteronbestimmungen im Blutplasma verfolgt.

\section{Experiment 2}

Basierend auf den aus dem ersten Experiment gewonnenen Daten erhielten je 10 Jungrinder an den Zyklustagen 8, 11 und 14 eine $\mathrm{PGF}_{2 \alpha}$-Applikation. Allen Tieren wurde 65 h später intramuskulär 0,05 mg GnRH (Gonavet ${ }^{\circledR}$, Berlin Chemie) verabreicht. Zusätzlich zu den Parametern, die im Experiment 1 erhoben wurden, wurden die Ovulationen im 6-h-Intervall durch rektale Untersuchungen bestimmt.

\section{Experiment 3}

In diesem Experiment wurden in verschiedenen landwirtschaftlichen Betrieben insgesamt 241 Jungrinder mittels $\mathrm{PGF}_{2 \alpha}$-Applikation synchronisiert. Alle Tiere waren klinisch gesund. Das Zyklusstadium der Tiere war auf Grund einer intensiven Brunstbeobachtung bekannt. Es wurden im Experiment 3A nur Tiere vom 11. bis 14. Zyklustag ausgewählt, bei denen auch durch rektale Untersuchung ein funktionelles Corpus luteum diagnostiziert wurde. Die Jungrinder wurden zufällig in eine Versuchs- bzw. Kontrollgruppe eingeordnet.

Tiere der Kontrollgruppe (BOB) wurden brunstorientiert besamt. Die Tiere der Versuchsgruppe (TOB) wurden 13 h nach Applikation von GnRH (65 nach der Luteolyseinduktion) terminorientiert besamt. In die Versuchsgruppe (TOB) vom Experiment 3B wurden Jungrinder vom Zyklustag 8 bis 14 eingeordnet. Alle Tiere dieser Versuchsgruppe wurden nach dem gleichen Regime wie in 3A behandelt. Als Kontrollgruppe dienten spontan brünstige Rinder (BOB).

Für Versuche an Hochleistungskühen wurden insgesamt 182 Tiere aus zwei weiteren landwirtschaftlichen Betrieben genutzt. Im Betrieb D wurden die Tiere der Versuchsund Kontrollgruppe nach dem gleichen Schema wie die Jungrinder behandelt. Die Luteolyse erfolgte im Zeitraum vom ZT 8 bis 14. Im Betrieb C wurden die Kühe der Kontrollgruppe (BOB) ohne vorherige Brunstsynchronisation brunstorientiert besamt. Die Tiere der Versuchsgruppen (TOB) wurden terminorientiert nach o.g. Schema behandelt und besamt.

Die Trächtigkeitsdiagnose wurde bei allen Tieren durch rektale Palpation zwischen dem 35. und 42. Trächtigkeitstag erstellt.

\section{Probenaufbereitung}

Über Venenverweilkatheter in der $V$. jugularis wurde den Tieren nach einem entsprechenden Entnahmeplan 8 bis $10 \mathrm{ml}$ Blut entnommen. Die Blutproben wurden mit einem Antikoagulans versehen. Durch Zentrifugation (10 Minuten bei $3000 \mathrm{U} / \mathrm{min}$ ) war eine Separation des Blutplasmas möglich, welches bis zur Hormonbestimmung bei $-18{ }^{\circ} \mathrm{C}$ gelagert wurde. 


\section{Hormonbestimmung}

Die Bestimmung der LH- und Progesteronkonzentrationen wurde mittels homologen Radioimmunoassay durchgeführt. Die Durchführung der Assays ist bei BLÖDOW (1988) und KANITZ et al. (1990) beschrieben. Der Intraassay-CV variiert zwischen 9 und $12 \%$; der des Interassays zwischen 11 und $14 \%$. Die Hormonkonzentrationen der einzelnen Tiere wurde in individuellen Assays bestimmt.

\section{Statistische Bearbeitung}

Für die Auswertung und statistische Bearbeitung wurde das Softwarepaket SAS ${ }^{\circledR}$ (System für Windows, 6.10) genutzt. Als statistische Maßzahlen wurden das arithmetische Mittel $(\bar{x})$, die Standardabweichung $(\bar{x} \pm \mathrm{s})$ und die Häufigkeitsverteilung (\%) mit Hilfe des Student`s t-Test berechnet. Analysen der Östrus- und Trächtigkeitsraten wurden mit Hilfe des Chi-square-Testes durchgeführt. Die Beeinflussung der Trächtigkeitsraten über die Einflussgrößen Besamungsregime, landwirtschaftlicher Betrieb und Bulle wurden mittels GLM-Analyse über das SAS ${ }^{\circledR}$ Programm (System für Windows, Release 6.10) berechnet.

\section{Ergebnisse}

Die Östrusrate aller Jungrinder betrug 90 - 100 \%. Die Brunst trat bei 94 \% der Jungrinder 48 - 72 Stunden nach der PGF $_{2 \alpha}$-Applikation auf. Die am 5. oder 8. Zyklustag einer Brunstinduktion unterzogenen Jungrinder reagierten schneller und synchroner auf die Verabreichung von $\mathrm{PGF}_{2 \alpha}$ als die am 11. oder 14. Zyklustag behandelten Tiere. Anhand der äußeren Brunstsymptome konnte nicht eindeutig entschieden werden, ob es sich um eine ovulatorische Brunst handelt. Die Östrusrate der Hochleistungstiere war mit 83,3 bis $87,5 \%$ gegenüber der bei Jungrindern vermindert.

Die ermittelten Progesteronkonzentrationen im Blutplasma z.Z. der Luteolyseinduktion wiesen eine gerichtete Beziehung zum Zyklustag auf und waren am Zyklustag 14 mit durchschnittlich 6,9 $\pm 0,9 \mathrm{ng} / \mathrm{ml}$ am höchsten. Am ZT 5 wurde mit 2,9 $\pm 0,6 \mathrm{ng} / \mathrm{ml}$ eine signifikant geringere Progesteronkonzentration gemessen. Innerhalb von 24 Stunden nach der $\mathrm{PGF}_{2 \alpha}$-Applikation sank die Progesteronkonzentration im Vergleich zum Ausgangswert um durchschnittlich 55 - 78 \% bei den am 8., 11. oder 14. Zyklustag behandelten Tieren. Eine Beeinflussung der luteolytischen Prozesse in Abhängigkeit vom erreichten Zyklusstand z. Z. der $\mathrm{PGF}_{2 \alpha}$-Injektion konnte bei diesen Jungrindern nicht ermittelt werden. Bei den am 5. Zyklustag in eine Brunstinduktionsbehandlung einbezogenen Tieren wurde nur bei $40 \%$ der Tiere an Hand der Progesteronkonzentrationsverläufe eine vollständige Luteolyse nachgewiesen. Ein LH-Peak und eine nachfolgende Ovulation wurde nur bei diesen Tieren ermittelt. Der LH-Peak trat bei Jungrindern, die am 5. oder 8. Zyklustag behandelt wurden, eher als bei den am 11 . oder 14. Zyklustag behandelten Tieren auf. Die durchschnittlichen Zeitintervalle von der PGF $_{2 \alpha}$-Injektion bis zum LH-Peak betrugen 57 bis 76 Stunden (Tab. 1).

Eine GnRH-Injektion 65 Stunden nach der PGF $_{2 \alpha}$-Applikation induzierte bei 56,7 \% der Jungrinder einen LH-Peak. Bei 43,3 \% der Jungrindern wurde der endogene LHPeak bereits 49 bis 64 Stunden nach der PGF $_{2 \alpha}$-Applikation festgestellt. Von den am 8. Zyklustag behandelten Tieren wiesen $90 \%$ einen endogenen LH-Peak auf. Bei den am 11. oder 14. Zyklustag mit PGF ${ }_{2 \alpha}$ behandelten Tieren induzierte die GnRH-Injektion bei 90 bzw. 70 \% der Jungrinder einen LH-Peak. 
Tabelle1

Zeitintervalle für klinische und endokrinologische Ereignisse nach der Applikation von PGF $2 \alpha$ (Mean Intervals between application of PGF $2 \alpha$ and LH-surge in heifers)

\begin{tabular}{cccc}
\hline ZT & $\begin{array}{c}\text { Tieranzahl } \\
\text { (gesamt) } \\
(\mathrm{n})\end{array}$ & $(\mathrm{n})$ & $\begin{array}{c}\text { Intervall PGF } 2 \alpha^{-A p p l . ~-~ L H-P e a k ~} \\
(\mathrm{~h})\end{array}$ \\
\hline 5 & 10 & 4 & $\bar{X} \pm \mathrm{s}$ \\
8 & 10 & 6 & $57,0 \pm 8,6^{\mathrm{a}}$ \\
11 & 11 & 9 & $62,7 \pm 12,7^{\mathrm{b}}$ \\
14 & 12 & 12 & $76,3 \pm 10,0^{\mathrm{c}}$ \\
\hline Kontrolle & 11 & 10 & $69,5 \pm 7,3^{\mathrm{d}}$ \\
gesamt & 54 & 41 & - \\
\hline a:c, a:d, b:c, c:d, & $\alpha<0.05$ & &
\end{tabular}

Eine GnRH-Applikation 65 Stunden nach PGF $_{2 \alpha}$ führte zur Terminierung des LHPeaks. Das durchschnittliche Intervall GnRH-Applikation bis zum induzierten LHPeak betrug 2 Stunden. Die Ovulationen traten 21 bis 23 Stunden nach dem LH-Peak auf (Tab. 2).

Tabelle 2

Zeitintervalle für klinische und endokrinologische Ereignisse nach der Applikation von PGF ${ }_{2 \alpha}$ und GnRH (Interval of clinical and endocrinological events after application of $\mathrm{PGF}_{2 \alpha}$ and $\mathrm{GnRH}$ )

\begin{tabular}{|c|c|c|c|c|c|c|c|}
\hline \multirow[t]{2}{*}{$\mathrm{ZT}$} & \multirow[t]{2}{*}{$\begin{array}{l}\text { Tier- } \\
\text { zahl } \\
\text { (n) }\end{array}$} & \multicolumn{2}{|c|}{$\begin{array}{c}\text { Intervall PGF }{ }_{2 \alpha^{-}} \\
\text {Applikation - LH-Peak } \\
\text { (h) }\end{array}$} & \multicolumn{2}{|c|}{$\begin{array}{c}\text { Intervall PGF } F_{2 \alpha} \text {-Applikation } \\
\text { - Ovulation } \\
\text { (h) }\end{array}$} & \multicolumn{2}{|c|}{$\begin{array}{c}\text { Intervall } \\
\text { LH-Peak - Ovulation } \\
\text { (h) }\end{array}$} \\
\hline & & (n) & $\bar{x} \pm \mathrm{s}$ & (n) & $\bar{x} \pm \mathrm{s}$ & (n) & $\bar{X} \pm \mathrm{s}$ \\
\hline 8 & 10 & 10 & $58,0 \pm 6,8^{\mathrm{a}}$ & 10 & $80,8 \pm 8,9^{c}$ & 10 & $22,8 \pm 7,8$ \\
\hline 11 & 10 & 10 & $66,2 \pm 2,4^{b}$ & 9 & $88,5 \pm \quad 0^{d}$ & 9 & $21,5 \pm 0$ \\
\hline 14 & 10 & 10 & $66,6 \pm 0,8^{\mathrm{b}}$ & 10 & $88,5 \pm \quad 0^{d}$ & 10 & $23,5 \pm 0$ \\
\hline Kontrolle & 4 & - & - & - & - & 4 & $22,9 \pm 5,6$ \\
\hline gesamt & 34 & 30 & & 29 & & 33 & \\
\hline
\end{tabular}

In Experiment 3 wurden insgesamt 241 Jungrinder und 182 Milchkühe einer Brunstsynchronisation unterzogen (Tab. 3 und 4). Die Jungrinder wiesen dabei tendenziell höhere Östrusraten als die Milchkühe auf. Die Trächtigkeitsrate nach terminorientierter Besamung war in allen 4 Teilexperimenten tendenziell höher als nach brunstorientierter Besamung.

Tabelle 3

Östrus- und Trächtigkeitsraten bei brunst- bzw. terminorientiert besamten Jungrindern (Estrous and pregnancy rates of heifers after insemination in heat or after timed insemination)

\begin{tabular}{lc|cccc}
\hline & & \multicolumn{2}{|c}{ Experiment A } & \multicolumn{2}{c}{ Experiment B } \\
& & TOB & BOB & TOB & BOB \\
\hline $\begin{array}{l}\text { Anzahl } \\
\text { synchronisierter Tiere }\end{array}$ & (n) & 108 & 107 & 26 & \\
$\begin{array}{l}\text { Anzahl Tiere mit Brunst } \\
\text { und KB }\end{array}$ & $(\mathrm{n})$ & 105 & 106 & 25 & 133 \\
Östrusrate & & & & & 95,0 \\
Anzahl tragender Tiere & $(\%)$ & 97,2 & 99,1 & 96,2 & 84 \\
Trächtigkeitsrate & $(\mathrm{n})$ & 83 & 74 & 19 & 63,1 \\
\hline
\end{tabular}

Aus Tabelle 5 wird ersichtlich, dass die verwendeten Bullen keinen Einfluss auf die erreichten Trächtigkeitsraten ausübten. Mit dem verwendeten Besamungsregime konnten in allen beteiligten landwirtschaftlichen Betrieben höhere Trächtigkeitsraten 
erreicht werden. Es wird aber auch deutlich, dass die Bedingungen in diesen Landwirtschaftsbetrieben die Trächtigkeitsrate signifikant beeinflussten.

Tabelle 4

Östrus- und Trächtigkeitsraten bei brunst- bzw. terminorientiert besamten Hochleistungskühen (Estrous and pregnancy rates of high yielding dairy cows after insemination in heat or after timed insemination)

\begin{tabular}{lc|cccc}
\hline & & \multicolumn{2}{|c}{ Experiment C } & \multicolumn{2}{c}{ Experiment D } \\
& & TOB & BOB & TOB & BOB \\
\hline $\begin{array}{l}\text { Anzahl } \\
\text { synchronisierter Tiere }\end{array}$ & (n) & 117 & & 18 & 47 \\
$\begin{array}{l}\text { Anzahl Tiere mit Brunst } \\
\text { und KB }\end{array}$ & 102 & 96 & & 40 \\
Östrusrate & $(\mathrm{n})$ & & & & \\
$\begin{array}{l}\text { Anzahl tragender Tiere } \\
\text { Trächtigkeitsrate }\end{array}$ & $(\mathrm{n})$ & 87,2 & & 83,3 & 85,1 \\
& $(\%)$ & 48 & 37 & 8 & 18 \\
\end{tabular}

Tabelle 5

Ergebnisse der GLM-Analyse $\left(\mathrm{SAS}^{\circledR}\right)$ bezüglich der Trächtigkeitsraten (Results of GLM-analysis (SAS ${ }^{\circledR}$ ) regading pregnancy rates)

\begin{tabular}{lcc}
\hline Parameter & F Value & Pr > F \\
\hline Besamungsregime & 1,28 & 0,2584 \\
Betrieb & 9,44 & 0,0024 \\
Besamungsregime * Betrieb & 0,01 & 0,9318 \\
Bulle (Betrieb) & 0,81 & 0,5176 \\
Besamungsregime *Bulle (Betrieb) & 0,14 & 0,9682 \\
\hline
\end{tabular}

\section{Diskussion}

Hohe Trächtigkeitsraten nach einer einmaligen terminorientierten Besamung erfordern Kenntnisse über Prozesse der Follikelentwicklung und Ovulation. Der LH-Peak ist der Impulsgeber für eine Vielzahl von Prozessen der Follikel- und Eizellreifung, der Ovulation sowie der Transformation der Granulosazellen in Luteinzellen (FORTUNE et al., 1988; DRIANCOURT, 1995; KANITZ et al., 1996; MIHM et al., 1994; KANITZ et al., 2003).

Ergebnisse aus dem ersten Experiment belegen eine große zeitliche Variation im Auftreten des LH-Peaks nach einer $\mathrm{PGF}_{2 \alpha}$-Applikation an den Zyklustagen 5 bis 14. Im Mittel variierte sein Auftreten zwischen 57,0 und 76,3 h post injectionem. Damit werden Erkenntnisse aus früheren Studien (TANABE und HANN, 1984; LARSON und BALL, 1992) bestätigt. Appliziert man $\mathrm{PGF}_{2 \alpha}$ in einer frühen Zyklusphase (ZT 5 bis 8) werden dominante Follikel der ersten Follikelreifungswelle indirekt beeinflusst. Das Intervall zwischen Luteolyseinduktion und LH-Peak bzw. Ovulation ist bei solchen Tieren kürzer. Die Kapazität zur Steroidsynthese der Follikel der ersten Follkelreifungswelle ist zu diesem Zeitpunkt hoch und über den positiven östrogenbedingten Feedbackmechanismus erfolgt innerhalb eines signifikant kürzeren Intervalls der LHPeak. Injektionen von $\mathrm{PGF}_{2 \alpha}$ in einer späteren Zyklusphase (ZT 11 bis 14) resultieren in einem längeren Intervall von der Applikation bis zur Auslösung des LH-Peaks. Im Regelfall trifft eine $\mathrm{PGF}_{2 \alpha}$-Injektionen am ZT 11 auf Follikel der zweiten Follikelreifungswelle bei denen der Leitfollikel zu diesem Zeitpunkt einen längeren Zeitraum bis zur Erlangung der funktionellen Dominanz benötigt, welches das längste mittlere Intervall zwischen Prostaglandinapplikation und LH-Peak erklärt (MILDNER und 
FREYMAN, 1992; BECKER, 1995; MIHM et al., 1997). Diese Follikel benötigen eine vergleichsweise längere Zeit, um Estradiol-17 $\beta$ in solchen Konzentrationen zu synthetisieren, die zur Auslösung des LH-Peaks erforderlich sind. Entsprechende Follikel ovulieren im Regelfall später. Bei den am ZT 14 behandelten Tieren leitet das $\mathrm{PGF}_{2 \alpha}$ die Luteolyse zu einem Zeitpunkt ein, an dem gereifte dominante Follikel der 2. Reifungswelle bereits zu einer gesteigerten Steroidsynthese befähigt sind und eher in der Lage sind den LH-Peak auszulösen. Das kürzeste durchschnittliche Zeitintervall von der Luteolyseinduktion bis zum LH-Peak wurde bei Tieren beobachtet, die am ZT 5 eine Prostaglandinapplikation erhielten. Da auf Grund einer unvollständigen Luteolyse nur 40 \% der Tiere dieser Gruppe mit einem LH-Peak reagierten, ist dieses Zyklusstadium für eine Zyklussynchronisation ungeeignet. In Übereinstimmung mit Ergebnissen anderer Studien (WATTS und FUQUAY, 1985; FORTUNE et al., 1988; FORTUNE, 1994; MARTINEZ et al., 2000) steigt der Anteil Tiere mit festgestelltem LH-Peak mit steigendem Zyklustag, an dem die Luteolyse induziert wurde, an.

Eine terminorientierte Besamung innerhalb von Tiergruppen kann nur erfolgreich sein, wenn ein gezieltes synchrones Auslösen des LH-Peaks möglich ist und wenn der Zeitpunkt der Freisetzung der Eizellen somit bekannt ist und der Parameter keine große Variation aufweist. Da die Ergebnisse aus dem Experiment 1 gezeigt haben, dass diese Voraussetzung nach alleiniger Gabe von $\mathrm{PGF}_{2 \alpha}$ nicht gegeben ist, bestand das Ziel des Experiments 2 darin, das Auftreten des LH-Peaks durch eine GnRH-Applikation 65 h nach Luteolyseinduktion zu synchronisieren.

Bei Jungrindern, bei denen am ZT 11 bzw. 14 die Luteolyse induziert wurde, wurde der LH-Peak im Mittel 66 h nach der PGF $_{2 \alpha}$-Injektion festgestellt. Die Standardabweichung war in beiden Tiergruppen gering (66,2 \pm 2,4 h bzw. 66,6 \pm 0,8 h). Bei 75 \% der Tiere wurde der LH-Peak innerhalb von 2 Stunden nach der GnRH-Applikation ermittelt. Bei den anderen Tieren, die bereits vor der GnRH-Behandlung einen LHPeak aufwiesen, wurde er 2 bis 6 h vor der Behandlung beobachtet. Erwartungsgemäß zeigte ein hoher Anteil Tiere aus der Versuchsgruppe ZT 8 bereits einen LH-Peak vor der Applikation von GnRH. Diese Ergebnisse belegen, dass bei Tieren der Versuchsgruppen ZT 11 und ZT 14 das Auftreten des LH-Peaks zeitlich vorverlagert und auf ein Intervall von ca. 67 h nach Luteolyseinduktion begrenzt werden konnte. Bei den Tieren, die am ZT 8 synchronisiert wurden, erfolgte der endogene LH-Peak in zeitlicher Nähe zum induzierten LH-Peak der Tiere vom ZT 11 und 14. Das gewählte Intervall von 65 h erwies sich somit als geeignet, die Mittelwertunterschiede für den Parameter Intervall $\mathrm{PGF}_{2 \alpha}$-Applikation bis zum Auftreten des LH-Peaks sowie die Varianzen für diesen Parameter zu minimieren. Das Intervall vom LH-Peak bis zur Ovulation war relativ uniform und unabhängig vom Tag, an dem die Luteolyse induziert wurde (21,5 bis 22,9 h).

Basierend auf diesen Ergebnissen wurden Besamungsversuche an Jungrindern und Kühen durchgeführt. Für die terminorientierte Besamung erhielten die Tiere 65 h nach der Luteolyseinduktion eine GnRH-Applikation. Die künstliche Besamung wurde unter Berücksichtigung der physiologischen Abläufe der Follikel- und Eizellreifung, der Ovulation sowie der Kapazitierung der Spermien und der Dauer der Befruchtungsfähigkeit der Spermien 13 h später durchgeführt.

Die erzielten Östrusraten sind ein Ausdruck für die Sicherheit der Luteolyseinduktion durch $\mathrm{PGF}_{2 \alpha}$-Präparate. Die mittels brunstorientierter Besamung erreichten Trächtigkeitsergebnisse von 69,8 \% und 63,1 \% zeugen von einer guten Fruchtbarkeitssituation 
in diesen Jungrinderherden. Die Steigerung der Trächtigkeitsraten nach terminorientierter Besamung um 9,2 bzw. 12,9 \% kann mit den vorliegenden Tierzahlen statistisch nicht gesichert werden. Sie unterstreicht aber, dass durch das gewählte Synchronisationsregime mit einer terminorientierten Besamung unabhängig vom Beginn und der Ausprägung der äußeren Brunstsymptomen hohe Trächtigkeitsraten realisierbar sind. Entsprechende Ergebnisse konnten nach Anwendung des beschriebenen Synchronisationsregimes bei Hochleistungskühen reproduziert werden. In beiden landwirtschaftlichen Betrieben konnte die Trächtigkeitsrate um 8,5 bzw. 8,3 \% gesteigert werden. Über ähnliche Ergebnisse nach Anwendung einer Prostaglandin - GnRH-Behandlung sowohl bei Milch- als auch bei Fleischrindern berichten u. a. AMBROSE et al., 2000; LAGO et al., 2000; AALI et al., 2001; RAJAMAHENDRAN et al., 2001; MACMILLAN et al., 2003 und EVANS et al., 2003. Bemerkenswert ist, dass in diesen internationalen Studien die Rinder nicht selektiert wurden. Alle Rinder einer Versuchsgruppe konnten durch entsprechende hormonelle Vorbehandlungen in das Synchronisationsregime einbezogen werden. Das für die o. g. Untersuchungen verwendete Schema besitzt demgegenüber Nachteile. Diese bestehen darin, dass es nicht unabhängig vom Zyklusstand der Tiere angewandt werden kann und dass die Zeiten für die Applikation der Arzneimittel bzw. die Besamung unter arbeitsorganisatorischen Aspekten z.T. nicht optimal in entsprechende Arbeitsabläufe zu integrieren sind. Nach deutschem Arzneimittelrecht sind zusätzliche hormonelle Vorbehandlungen (Kombinationen mit Östradiol und/oder Progesteron) nicht zulässig. Auf der Basis zugelassener Präparate ermöglicht das verwendete Behandlungsregime deshalb auch in leistungsstarken Milchviehherden das Reproduktionsmanagement effizienter zu gestalten.

\section{Schlussfolgerungen}

Die in den eigenen Untersuchungen erzielten Östrus- und Trächtigkeitsraten beweisen, dass das gewählte Behandlungs- und Besamungsregime auf der Basis einer kombinierten Prostaglandin- und GnRH-Behandlung geeignet war, ein konzentriertes Auftreten von Östrus, LH-Peak und Ovulation und somit eine Brunst- und Ovulationssynchronisation bei den am 8. bis $14 \mathrm{ZT}$ behandelten Tieren zu erreichen.

Der mit 13 h nach GnRH bzw. 78 h nach der Prostaglandinapplikation festgelegte Zeitpunkt der terminorientierten Besamung entsprach den physiologischen Abläufen der Follikel-, Eizell- und Spermienreifung und ermöglicht hohe Trächtigkeitsraten.

\section{Literatur}

AALI, M.A.; SMALL, J.A.; CHENG, K.M.; RAJAMAHENDRAN, R.:

In vitro assesment of the corpus luteum function in cattle following OVSYNCH and CIDR estrus synchronization protocols. (2001) Proc. $34^{\text {th }}$ ISAR-meeting, 145

AMBROSE, D.J.; KASTELIC, J.P.; RAJAMAHENDRAN, R.; SMALL, J.A.; URTON, G.:

Pregnancy rates in dairy cows after GnRH-treatment at 7, 14, or 7 and 14 days after timed AI. Can. J. Anim. Sci. 80 (2000), 755

AMBROSE, D.J.; RAJAMAHENDRAN, R.; KASTELIC, J.P.; SMALL, J.A.:

Synchronization of ovulation and conception rates in Holstein heifers given an intravaginal progesterone device (CIDR) and estradiol cyprionate, porcine LH or gonadotropin releasing hormone. Arch. Tierz., Dummerstorf 44 (2001) Special Issue, 77-79

BECKER, F.:

Untersuchungen zur Follikelentwicklung und zur Luteolyse nach $\mathrm{PGF}_{2 \alpha}$-Applikation an verschiedenen Zyklustagen beim Rind. Vet. Med. Diss., FU Berlin (1995) 
BLÖDOW, G.; GÖTZE, M.; KITZIG, M.; BRÜSSOW, K.; DUSCHINSKI, U.:

Radioimmunologische Steroidhormonbestimmungenin der Follikelflüssigkeit bei Rind und Schwein. Isotopenpraxis 24 (1988), 151 -155

BOHJWANI, S.; BECKER, F.; KANITZ, W.:

Studying the efficacy of a combination of GnRH and Prostaglandin treatment for estrus synchronization in Holstein heifers. Arch. Tierz., Dummerstorf 44 (2001) Special Issue, 161

CARTMILL, J.A.; EL-ZARKOUNY, S.Z.; HENSLEY, B.A. ; LAMB, G.C., STEVESON, J.S.:

Stage of estrous cycle, incidence and timing of ovulation and pregnancy rates in dairy cattle after three timed breeding protocols. J. Dairy Sci. 84 (2001), 1051-1059

DRIANCOURT, M.A.:

Endocrine and local control of follicular growth and maturation. Reprod. Dom. Anim. 30 (1995), 309-317

EVANS, A.C.; KUFFE, O.; MIHM, M.; ROCHE, J.F.; MACMILLAN, K.L.; BOLAND M.P.:

Effect of oestradiol benzoatgiven after prostaglandinat two stagesof follicular wavedevelopmenton oestrus synchronisation, the LH surge and ovulation in heifers. Anim. Reprod. Sci. 76 (2003), 13-23

FORTUNE, J.E.; SIROIS, J.; QUIRK, S.M.:

The growth and differentiationof ovarian follicles during the bovine estrous cycle. Theriogenology 29 (1988), 95-107

FORTUNE, J.E.:

Ovarian follicular growth and development in mammals. Biol. Reprod. 50 (1994), 225-32

KANITZ, W.; MILDNER, H.; FREYMANN, U.; KANITZ, E.; SCHNEIDER, F.; BECKER, F.:

Untersuchungen zum Ovulationszeitraum nach induzierter Luteolyse beim Rind in Abhängigkeit vom Ovulationszeitraum. Züchtungskunde 68 (1996), 268-279

KANITZ, W.; BECKER, F.; DIETL, G.; REINSCH, N.; STAUFENBIEL, R.:

Beziehungen zwischen Milchleistung, Energieversorgung und Fruchtbarkeit unter den Bedingungen von Hochleistung beim Rind. Züchtungskunde 75 (2003), 489-498

KANITZ, E.; SCHNEIDER, F.; KANITZ, W.; KITZIG, M.:

Erste Ergebnisse eines RIA für das LH zur Kontrolle der Superovulation beim Rind. Archiv exper. Vet. med. 39 (1990), 901- 909

LAGO, I.; ARMAND, U.; VELASQUES, M.; PIAGGIO, J.:

Comparison of three different estrus synchronization treatments with and without "set time" insemination. Theriogenology 53 (2000), 200-202

LARSON, L.L.; BALL, P.J.H.:

Regulation of estrous cycle in dairy cattle: A review. Theriogenology 38 (1992), 255-267

MACMILLAN, K.L.; SEQWAQWE, B.V.; PINO, C.S.:

Association between the manipulation of pattern of follicular development and fertility in cattle. Anim. Reprod. Sci. 78 (2003), 327-344

MARTINEZ, M.F.; ADAMS, G.P.; KASTELIC, J.P.; BERFELDT, D.R.; MAPLETOFT, R.J.:

Induction of follicular wave emergence for estrous synchronization and artificial insemination in heifers. Theriogenology 54 (2000), 757-769

MIHM, M.; BAGUSI, A.; BOLAND, M.P.; ROCHE, J.F.:

Association between the duration of dominance of the ovulatory follicle and pregnancy rates in beef heifers. J. Reprod. Fertil. 102 (1994), 123-130

MILDNER, H.; FREYMANN, U.:

Untersuchungen zur Luteolyse und zur Ovulation nach Prostaglandinapplikation allein sowie in Kombination mit GnRH an verschiedenen Zyklustagen beim Rind. Vet. Med. Diss. Leipzig (1992)

MOREIRA, F.; DE LA SOTA, R.L.; DIAZ, T.; THATCHER W.W.:

Effect of day of the estrous cycle at the initiation of a timed artificial insemination protocol on responses in dairy heifers. J. Anim. Sci. 78 (2000), 811-819

PETERS, M.W.; PURSLEY, J.R.:

Timing of final GnRH of the Ovsynch protocol affects ovulatory follicle size, subsequent luteal function, and fertility in dairy cows. Theriogenology $\mathbf{6 0}$ (2001) 6, 1197-204

PURSLEY, J.R.; MEE, M.O.; WILTBANK, M.C.:

Synchronization of ovulation in dairy cows using PGF $_{2 \alpha}$ and GnRH. Theriogenology 44 (1995), 915-923

PURSLEY, J.R.; WILTBANK, M.C.; OTTOBRE, J.S.; BARVERICK, H.A.; ANDERSON, L.L.:

Pregnancy rates per artificial insemination for cows and heifers inseminated at a synchronized ovulation or synchronized estrus. J. Dairy Sci. 80 (1997), 295-300

RAJAMAHENDRAN, R.; AMBROSE, D.J.; SMALL, J.A.; DINN, N.:

Synchronization of estrus and ovulation in cattle. Arch. Tierz., Dummerstorf 44 (2001) Special Issue, 5867

SCHMITT, E.J.P.; DIAZ, T.; DROST, M.; THATCHER, W.W.:

Use of a gonadotropin-releasing hormone agonist or hCG for timed insemination in cattle. J. Anim. Sci. 74 (1996), 1084-1091 
TWAGIRAMUNGU, H.; GUIBAULT, L.A.; DUFOR, J.J.:

Synchronization of ovarian follicular waves with gonadotropin releasing hormone agonist to increase the precision of estrus in cattle: a review. J. Anim. Sci. 73 (1995), 3141-3151

TANABE, T.Y.; HANN, R.C.:

Synchronized estrus and subsequent conception in dairy heifers treated with prostaglandin F. 1. Influence of stage of cycleat treatment. J. Anim. Sci. 58 (1984), 805-811

WATTS, T.L.; FUQUAY, J.W.:

Response and fertility of dairy heifers following injection of prostaglandin during early, middle and late diestrus. Theriogenology 23 (1985), 655-661

Eingegangen: 26.11.2003

Akzeptiert: 17.12.2003

Autor für Korrespondenz

Dr. FRANK BECKER

Forschungsinstitut für die Biologie landwirtschaftlicher Nutztiere

Forschungsbereich Fortpflanzungsbiologie

Wilhelm-Stahl-Allee 2

D-18196 Dummerstorf

E-Mail: becker@fbn-dummerstorf.de 